\title{
Structure of a Novel Rubisco Activase in Gardenia jasminoides
}

\author{
Lan Gao \\ Guangdong Pharmaceutical University, Guangzhou Higher Education Mega Center \\ Guangzhou 510006, Peoples Republic of China \\ glgdpu@aliyun.com
}

\begin{abstract}
Keywords: Rubisco; Rubisco activase; Gardenia jasminoides; cDNA library; 3D-structure. Abstract. The key photosynthetic $\mathrm{CO}_{2}$-fixing enzyme ribulose-1,5-bisphosphate carboxylase/ oxygenase (Rubisco) is regulated by a protein known as Rubisco activase (RCA). RCA is a soluble chloroplast ATPase encoded by nuclear genome that catalyzes the activation of Rubisco in vivo by the ATP-dependent removal of various inhibitory sugar phosphates. In this work, we constructed a G.jasminoides fruit cDNA library, and the GjRCA cDNA was isolated from the library by sequencing method. The GjRCA cDNA contains a predicted 1428 bp open reading frame that encodes 475 amino acids. A bioinformatics analysis was conducted with previously characterized RCAs from other plant species, the results suggested that the GjRCA amino acid sequence has highly conserved nucleotide-binding region, critical ATPase amino acid residue and Rubisco-interaction region with other RCAs. A three-dimensional monomer model of GjRCA was built, GjRCA has extended carboxyl terminus sequence with two conserved cysteine residues, and has $46 \mathrm{kD}$ molecular weight, suggest it's a L-isoform of RCA, it's similar to structure of AAA+ family protein and other RCAs. Conclusion: The results indicated that GjRCA is a L-isofrom RCA of G. jasminoides.
\end{abstract}

\section{Introduction}

Rubisco (Ribulose-bis-bisphosphate carboxylase/oxygenase) catalyzes carboxylation of RuBP in the first step of a series of reactions leading to the incorporation of atmospheric $\mathrm{CO}_{2}$ into biomass. To be active, a conserved active-site Lysine residue on the Rubisco large subunit must be carbamylated by a non-substrate $\mathrm{CO}_{2}$, followed by coordinated of a $\mathrm{Mg}^{2+}$ prior to binding RuBP. Ribulose-bis-phosphate carboxylase/oxygenase activase (RCA), first found by Someville [1], catalyzes the activation of Rubisco in vivo by the ATP-dependent removal of various inhibitory sugar phosphates, including RuBP from the nonactivated active site of Rubisco, thereby facilitating carbamylation and $\mathrm{Mg}^{2+}$ binding. In some cases, fully activated (metal-bound) Rubisco may bind inhibitory sugar phosphates that mimic the carboxylation transition state. These molecules are also removed by Rubisco activase to restore a functional active site [2]. Plants lacking activase or having a very low level of activase cannot survive at atmospheric $\mathrm{CO}_{2}$ levels, and those expressing reduced levels exhibit reduced rates of photosynthesis and growth [3]. Rubisco activase was identified as a member of a superset of the AAA+ protein family (ATPases associated with diverse cellular activities) based on sequence and structural homologies.

In many plant, there are two closely related forms of RCA proteins, a large $45-46 \mathrm{kD}$ isoform (L) and a small 41-43 kD isoform (S). Both the large and small isoforms are active in ATP hydrolysis and Rubisco activation. Genomic analyses have identified one RCA gene in spinach, Arabidopsis, rice and wheat [4], in which alternative splicing of the RCA transcript results in two RCA isoforms. Two RCA genes encode two RCA isoforms in barley, cotton and sweet potato (Ipomoea batatas) [5-6]. In barley, one alternatively spliced RCA gene (rcaA) produces two RCA isoforms, and the second gene (rcaB) encodes only the small isoform of RCA [5]. In tobacco and soybean, there are three RCA genes but only produce two small RCA isoform [7]. The greatest difference between the two RCA forms is at the 20- to 30-amino acid extension at the carboxyl terminus of the longer L-isoform. Compared with the small isoform, the large isoform has a carboxy-terminal extension that contains two redox-sensitive cysteine (Cys) residues [7]. The two Cys residues are conserved in L-isoform from diverse species, including those produced from separate genes. 
The activity of RCA is dependent on the ATP/ADP ratio [8] and/or the redox state of the chloroplast [9] and is sensitive to high temperature [10]. Light modulation of rubisco in Arabidopsis requires redox regulation of the large isoform. When these residues are oxidized to a disulfide, the affinity for ATP decreases and enzyme activity is more sensitive to inhibition by ADP. Thus, RCA can adjust the rate of $\mathrm{CO}_{2}$ fixation to the rate of electron transport activity and can limit $\mathrm{CO}_{2}$ assimilation during heat stress [11]. Studies on rice and spinach demonstrated that the L-isoform was more thermostable than the S-isoform, suggesting that the L-RCA isoform may play an important role in photosynthetic acclimation to moderate heat stress in vivo, whereas the S-RCA isoform was shown to play a major role in maintaining Rubisco's initial activity under normal conditions [12].

Rubisco and RCA do not form a stable binary complex, the physical interaction details is not elucidated [13]. The data indicate that activation of Rubisco by RCA involves altering the positions of specific domains around the Rubisco active site to allow bound sugar-phosphates to dissociate more rapidly [14]. A model for the mechanism of RCA action as follows [2], RCA is bound to Rubisco through electrostatic and other forces by the active site in the senser-2 (Sensor-2, a specific C-terminal domain in AAA+ protein for substrate recognition) domain; the interaction cause Rubisco moves accordingly, the bound sugar phosphate is free to dissociate and being activated.

Gardenia jasminoides originates in Asia and has been in cultivation for at least a thousand years. The fruit of G. jasminoides is used in Asian countries as a natural colorant, and as a traditional herbal medicine. In this paper, we identified and analyzed a Ribulose-bis-phosphate carboxylase/oxygenase activase (GjRCA) in G. jasminoides.

\section{Materials and Methods}

Plant and Growth Conditions. G.jasminoides plants cultivated at Guangdong Pharmaceutical University were used as materials. Fruits were collected at development stageII, closed with yellowish green exocarp and orange mesocarp. The samples were stored at $-80^{\circ} \mathrm{C}$ until required.

CDNA Library Construction, ESTs Sequencing and Cloning of GjRCA. Total RNA was extracted from Gardenia fruit (stageII), using a modified CTAB (hexadecyl trimethyl ammonium bromide) based extraction protocol [15]. From total RNA, the cDNA library construction and amplification were performed following the users manual of the Creator ${ }^{\mathrm{TM}}$ SMARTTM cDNA Library construction Kit (Clontech, USA). The SMART cDNAs were ligated into SfiI-digested pDNR-LIB vector and transformed into Escherichia coli strain DH5 $\alpha$. Colonies were randomly picked, inoculate each colony to a separated PCR reaction solutions. The colony was lysised by heating the mixed solutions at $95^{\circ} \mathrm{C}$ in a PTC-200 Thermocycler (MJ Research, USA) for 5 min. After then, went to PCR amplification procedure with M13 primers provided by the CreatorTM SMARTTM cDNA Library construction Kit. The amplified PCR products (ESTs, expressed sequence tags) were analyzed by $1.2 \%$ agarose gel electrophoresis. When the amplified PCR products were longer than $1000 \mathrm{bp}$, incubated the isolated colonies and sequenced the ESTs. There are $40 \mathrm{ESTs}$ were sequenced. After sequencing and analysis, the colony containing the predicted pDNR-LIB-GjRCA was isolated.

\section{Results and Discussion}

We identified novel RCA homologues in Gardenia jasminoides (named GjRCA) by exploiting the fruit cDNA library of $G$. jasminoides. The full-length GjRCA cDNA (Genbank accession No. KM371240) was obtained. The cDNA contains a predicted 1428 bp ORF that encodes 475 amino acids. The deduced amino acid sequence of GjRCA was compared with Genbank database, the best homology was found to RCA like protein of Coffea canephora. The two proteins share $92 \%$ identical amino acids. And compared GjRCA to the RCA proteins from six plant species. The GjRCA has a carboxy-terminal extension that contains two cysteine (Cys) residues; resemble RCAL-isoform; and contains high two nucleotide-binding region P-loop (sequence A: 166-173) and sequence B (226-231); hightly conserved critical ATPase residue R297 ('Arg finger'), R300, R350, R352; and similar Rubisco-interaction region with RCAs from other plants. It was predicted by online chlorop v1.1 
software that the GjRCA gene encodes a 57 amino acids long N-terminal transit peptide. RCA is preditated bound to Rubisco through active site involve 372-375 (GjRCA) residues KR (K) LV, it's highly conserved in a wide range of plants except Solanaceae family; ATP hydrolysis promotes movement of the C-terminal sensor-2 domain (350-386 on GjRCA); the interaction cause Rubisco moves accordingly, the bound sugar phosphate is free to dissociate and being activated. The GjRCA secondary structure was predicted by Swiss-model software.

The 3D (three dimension) structure of GjRCA was predicted using SWISS-Model Workspace. The X-Ray diffraction at resolutions down to $2.9 \AA$ of Arabidopsis thaliana RCAL-isoform (PBD code 4W5W) was used as template [16] (Fig. 1B). It has a classical AAA+ protein two-domain architecture. The RCA includes residues 65-362 (with 25 residues not visible in the structure), has $85 \%$ amino acid identity with GjRCA (residues 124-421). The structure was successfully built as a monomer (Fig. 1A); the fold of GjRCA consists of $\alpha / \beta$ subdomain (N-domain) with five $\alpha$-helices flanked by six-stranded parallel $\beta$-sheet, and $\alpha$-helical subdomain (C-domain). The protein packs in fashion as observed for other Rubisco activase [17]. Indicating a high level of structural similarity with Arabidopsis thaliana RCAL and identifies the enzyme as a member of the AAA+ family. The two nucleotide-binding region P-loop (166-173) and sequence B (226-231); critical ATPase residue R297 ('Arg finger'), R300, R350, R352; and Rubisco-interaction region (Sensor-2, 350-386), and the active site were shown. In Fig. 1C and Fig. 1D is a 3.6- $\AA$ X-ray structure of ring-shaped Vps4 (vacuolar protein sorting 4 AAA-ATPase) from Metallosphera sedula (MsVps4) complex with ADP (PDB Code 4d81). The Vps4 includes residues 97-363, has 17\% amino acid identity with GjRCA (residues 123-403). Compare the binding site of ATP, the structure suggests that the ATP-binding domain and the position of ATPase amino acid are similar with GjRCA.

A

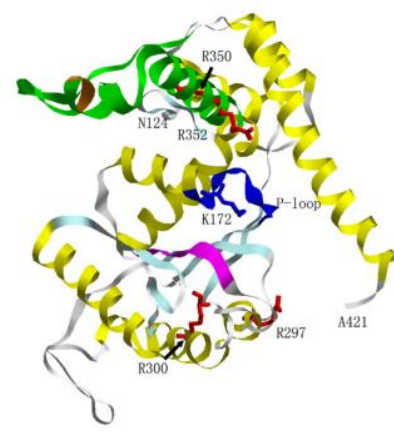

C

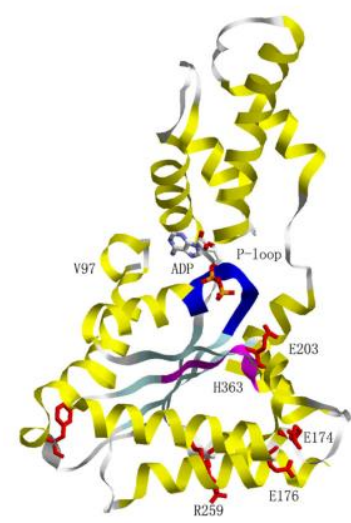

B

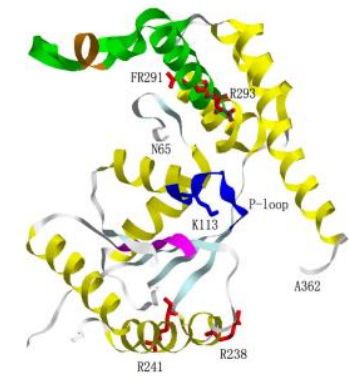

D

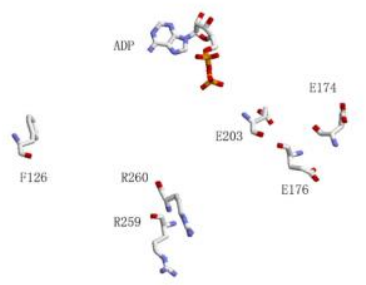

Figure 1. 3D model of GjRCA. (A): Model structure of GjRCA (124-421 residues; Genbank accession no. AIX10948), predited by Swiss-Model Workspace. (B): Crystal structure of Arabidopsis thaliana Rubisco activase (65-134, 145-172,183-202 and 214-362 residues), PBD code: 4W5W. (C): Crystal structure of the vacuolar protein sorting AAA-ATPase (Vps4) protein complex with ADP from Metallosphera sedula (97-363 residues), PBD code: 4D81. (D): The critical residues and ADP in (C) were shown in stick diagram. In (A-C): Shown in ribbon diagram, alpha helixes shown in yellow color, beta sheets are shown in cyanic color. P-loop and the second ATP binding domain were shown in blue and pink respectively. Active site was shown in brown. The region interaction with Rubisco was shown in green. Critical residues with ATP binding function shown in blue (A and B) or red (C) in stick diagram, and with ATPase function were shown in red. 
The number of RCA-encoding genes varies depending on the plant species. Activase from plants in Solanaceae family was shown to be a poor activator of Rubisco from a wide range of plants outside the Solanaceae family. From the sequence compare, we found the GjRCA has the same active site with non-solanaceous higher plants (KR (K) LV instead of DKLL).

\section{Summary}

Rubisco activase plays an important role in regulating plant photosynthesis and plant growth, which catalyses the activation of the key photosynthesis enzyme Rubisco, has been identified as one of several new potential targets for improving plant productivity and seed yield [18]. Genetic engineering experiments have been carried out to improve RCA activity [19]. This work suggests that, GjRCA has highly conserved ATP binding domain, ATPase amino acid, Rubisco interaction domain, active site with RCAs from other plant, and extended C-terminal; suggest it's a L-isoform of RCA.

\section{References}

[1]M.E.Salvucci, J.M.Werneke and W.L. Ogren et al: Plant Physiol. Vol. 84 (1987), p. 930-936

[2]A.R.Jr,Portis, C.Li and D.Wang et al: J Exp Bot. Vol. 59 (2008), p. 1597-1604

[3]S.von.Caemmerer, L.Hendrickson and V.Quinn et al: Plant Physiol. Vol. 137 (2005), p. 747-755

[4]K.Y.To, D.F.Suen and S.C.Chen: Planta. Vol. 209 (1999), p. 66-76

[5]S.J.Rundle and R.E.Zielinski: J Biol Chem. Vol. 266 (1991), p. 14802-14807

[6]Y.Jiang, J.Wang and X.Tao et al: Mol Biol Rep. Vol. 40 (2013), p. 6309-6321

[7]A.R.Jr.Portis: Photosynth Res. Vol. 75(2003), p. 11-27

[8]A.E.Carmo-Silva and M.E.Salvucci: Plant Physiol. Vol. 161(2013), p. 1645-1655

[9]D.Wang and A.R.Jr.Portis: J Biol Chem. Vol. 281(2006), p. 25241-25249

[10]D.Shivhare and O.Mueller-Cajar: Plant Physiol. Vol. 174 (2017), p. 1505-1516

[11]M.Chao, Z.Yin and D Hao et al: J Exp Bot. Vol. 65 (2014), p. 47-59

[12]D.Wang, X.F.Li and Z.J.Zhou et al: Physiol Plant. Vol. 139 (2010), p. 55-67

[13]J.C.Scales, M.A.Parry and M.E.Salvucci: Photosynth Res. Vol. 119 (2014), p. 355-365

[14]R.M.Wachter, M.E.Salvucci and A.E.Carmo-Silva et al: Photosynth Res. Vol. 117 (2013), p. 557-566

[15]I.Bekesiova, J.P.Nap and L.Mlynarova: Plant Mol. Biol. Rep, Vol. 17 (1999), p. 269-277

[16]D.Hasse, A.M.Larsson and I.Andersson: Acta Crystallogr D Biol Crystallogr. Vol. 71 (2015), p. 800-808.

[17]M.Stotz, O.Mueller-Cajar and S.Ciniawsky et al: Nat Struct Mol Biol. Vol. 18 (2011), p. 1366-1370

[18]J.A.Perdomo, S.Capó-Bauçà and E.Carmo-Silva et al: Front. Plant Sci.Vol. 8 (2016): 490.

[19]E.Carmo-Silva, J.C.Scales and P.J.Madgwick et al: Plant Cell Environ. Vol.38 (2015), p. 1817-1832 